\title{
Corantes naturais: pigmentos no ensino de Química
}

\author{
Natural dyes: pigments in Chemistry teaching \\ Colorantes naturales: pigmentos en la enseñanza de la Química
}

Recebido: 07/09/2021 | Revisado: 12/09/2021 | Aceito: 15/09/2021 | Publicado: 16/09/2021

Rothchild Sousa de Morais Carvalho Filho

ORCID: https://orcid.org/0000-0002-7067-6159

Universidade Estadual do Piauí, Brasil

E-mail: rothchildquimicahsb@gmail.com

Valdiléia Teixeira Uchôa

ORCID: https://orcid.org/0000-0001-8080-6335 Universidade Estadual do Piauí, Brasil E-mail: vtuquimica@yahoo.com.br

Natasha Alves Rocha

ORCID: https://orcid.org/0000-00003-1337-2885 Universidade Estadual do Piauí, Brasil

E-mail: natashalvesrocha@gmail.com

João Clécio Alves Pereira

ORCID: https://orcid.org/0000-0001-8915-0395 Instituto de Química de São Carlos, Brasil E-mail: clecioquimica@gmail.com

Naiana Machado Pontes

ORCID: https://orcid.org/0000-0001-9997-542X Universidade Estadual do Piauí, Brasil

E-mail: naianapontes6@gmail.com

Fernanda Meneses Amaral

ORCID: https://orcid.org/0000-0001-6439-6524 Universidade Estadual do Piauí, Brasil

E-mail: fenetonanda@gmail.com

Ana Kerly Oliveira de Brito

ORCID: https://orcid.org/0000-0002-3661-0359

Universidade Estadual do Piauí, Brasil

E-mail: anakerlybrito@gmail.com

Maria dos Remédios Brito Nascimento

ORCID: https://orcid.org/0000-0001-9223-4442 Instituto Federal de Educação, Ciência e Tecnologia do Piauí, Brasil E-mail: remeddynhabritto62@gmail.com

Denise Ribeiro Magalhães de Sousa Cerqueira ORCID: https://orcid.org/0000-0003-1084-3338 Instituto Federal de Educação, Ciência e Tecnologia do Piauí, Brasil

E-mail: denisemagalhaespaz@ hotmail.com

\section{Resumo}

Considerando que a utilização de itens presentes no cotidiano dos alunos é reconhecidamente uma estratégia adequada para transmissão e fixação do conhecimento. Neste contexto, os corantes naturais podem ser explorados didaticamente no ensino de Química, desde a obtenção até a caracterização visual. Os indicadores naturais ácido-base são corantes extraídos de partes de plantas (flores, frutos, folhas e galhos) com a presença de cromófaros. Esses cromófaros são responsáveis pela variação de coloração em função do pH. Dentre os cromófaros, podemos destacar as antocianinas, maior grupo de pigmentos naturais. O objetivo desse trabalho é apresentar alguns corantes naturais com potencialidade para o ensino de Química, almejando a contribuição das possibilidades de materiais alternativos para o ensino em diversos contextos regionais. Os dados apresentados demonstraram a diversidade de materiais, os quais se mostraram eficientes no uso como indicador, vindo a ser excelentes corantes naturais de fácil acesso e baixo custo para serem utilizados no ensino de Química.

Palavras-chave: Indicador ácido-base; Corantes naturais; Ensino de Química.

\begin{abstract}
Considering that the use of items present in the daily lives of students is recognized as an adequate strategy for transmitting and fixing knowledge. In this context, natural dyes can be explored didactically in teaching Chemistry from obtaining to visual characterization. Acid-base natural indicators are dyes extracted from chromophore-rich plant parts. These chromophares are responsible for the variation in color as a function of $\mathrm{pH}$. Among the
\end{abstract}


chromophares, we can highlight anthocyanins, the largest group of natural pigments. The objective of this work is to present some natural dyes with potential for the teaching of Chemistry, aiming at the contribution of the possibilities of alternative materials for teaching in different regional contexts. The data presented demonstrated the diversity of materials, which proved to be efficient in use as an indicator, being excellent natural dyes with easy access and low cost to be used in teaching Chemistry.

Keywords: Acid-sase indicator; Natural dyes; Chemistry teaching.

\section{Resumen}

Considerando que el uso de elementos presentes en la vida diaria de los estudiantes se reconoce como una estrategia adecuada para transmitir y fijar conocimientos. En este contexto, los tintes naturales pueden explorarse didácticamente en la enseñanza de la Química desde la obtención hasta la caracterización visual. Los indicadores naturales ácido-base son tintes extraídos de partes de plantas ricas en cromóforos. Estos cromófares son responsables de la variación de color en función del pH. Entre los cromófares, podemos destacar las antocianinas, el mayor grupo de pigmentos naturales. El objetivo de este trabajo es presentar algunos tintes naturales con potencial para la enseñanza de la Química, buscando el aporte de las posibilidades de materiales alternativos para la enseñanza en diferentes contextos regionales. Los datos presentados demostraron la diversidad de materiales, los cuales demostraron ser eficientes en su uso como indicador, siendo excelentes tintes naturales de fácil acceso y bajo costo para ser utilizados en la enseñanza de la Química.

Palabras clave: Indicador ácido-base; Tintes naturales; Enseñanza de la Química.

\section{Introdução}

No ensino de Química as aulas experimentais proporcionam ao aluno uma visão de como esse conhecimento se forma e se desenvolve, observando as transformações que ocorre em cada experimento (Azambuja, Goi \& Hartmann, 2021).

O ensino de forma apenas tradicional, com resolução de exercícios recomendadas pelos docentes, impede que os alunos, relacionem os conhecimentos adquiridos em sala com acontecimentos do seu cotidiano (Dias, Guimarães \& Merçon, 2003).

A utilização de experimentos é de suma importância, e a disciplina de Química é uma ciência experimental, de verificação científica, articulada a suposições teóricas, e de tal modo o conceito da concretização de experimentos é defendido como ampla tática didática para o ensino e aprendizagem dos estudantes (Penaforte \& Santos, 2014; Silva \& Lopes, 2021).

O uso de itens presentes no cotidiano dos alunos é reconhecidamente uma estratégia adequada para transmissão e fixação do conhecimento, tendo em vista que estas estratégias são priorizadas pela a Lei das Diretrizes e Bases da Educação (LDB) (Couto, Ramos \& Cavaleiro, 1998;). Neste contexto, corantes extraídos de partes das plantas são ricos em cromóforos capazes de mudar de cor em função do pH (Skoog et al., 2015; Uchôa et al., 2016; Penaforte \& Santos, 2014; Silva et al., 2020).

A utilização dos corantes pode ser explorada didaticamente, desde a etapa de obtenção até a estabilidade térmica, observando as mudanças de coloração no meio e degradação térmica dos corantes. (Uchôa et al., 2016; Soares, Silva \& Cavalheiro, 2001). Os extratos naturais vêm sendo utilizados frequentemente como indicadores naturais ácido-base, como alternativa aos indicadores sintéticos (Couto, Ramos \& Cavaleiro, 1998; Silva et al., 2020).

Os indicadores naturais são soluções fracas; ou seja, esse corantes apresentam um valor de pH próximo ao pH neutro, e quando adicionados a uma solução, eles ligam-se a íons $\mathrm{H}^{+}$ou $\mathrm{OH}^{-}$, apresentando variação de cor devido a uma alteração na configuração eletrônica (Carvalho Filho \& Pereira, 2016; Uchôa et al., 2016; Silva et al., 2020).

A ligação que ocorre com estes íons provoca alteração na configuração eletrônica dos corantes, tal alteração deve ser um dos motivos responsáveis pela variação de coloração presente no meio (Uchôa et al., 2016; Coelho, A. G. (2011). O valor de pH é o ponto de maior influência na variação de cor desses corantes apresentado pelas antocianinas, haja visto, que em função de sua acidez ou basicidade, essa substância pode apresentar diferentes estruturas (Lee, Durts \& Wrolstad, 2005). 
Em 1775, Bergman escreveu que extratos de plantas azuis são mais sensíveis aos ácidos, tendo em vista, que esses possuem uma variação gradual de cor em faixa de $\mathrm{pH}$, que pode diferenciar ácidos fracos de fortes e bases fracas de bases fortes (Harborne \& Grayer, 1988).

Logo, o objetivo desse trabalho é apresentar alguns corantes naturais com potencialidade para o ensino de Química, almejando a contribuição das possibilidades de materiais alternativos para o ensino em diversos contextos regionais.

\section{Metodologia}

\section{Obtenção da matéria prima}

As amostras foram colhidas em Piracuruca - PI, Brasil. Em seguida, foram levadas para o laboratório de Química da Universidade Estadual do Piauí - UESPI, campus Piripiri - PI.

\section{Preparo do Indicador e das Soluções}

As amostras dos corantes foram separadas de forma mecânica, lavadas em água corrente e pesadas (10g). As amostras em estudo foram tratadas com $50 \mathrm{~mL}$ de água destilada por maceração e decocção durante 15 minutos para obter-se um extrato aquoso. Após esse evento o material resultante foi filtrado. O procedimento de extração dos corantes pode ser observado na Figura 1 (Carvalho Filho \& Pereira, 2021).

Figura 1: Procedimento de obtenção do extrato.
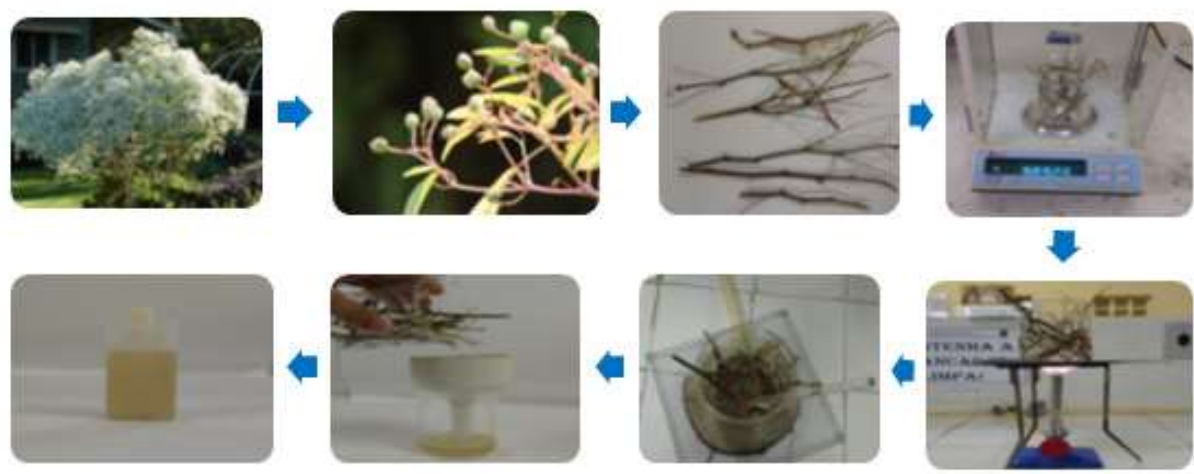

Fonte: Carvalho Filho \& Pereira, (2021).

Após o preparo dos extratos, avaliou-se a eficácia dos corantes em soluções ácidas e básicas. Todas as soluções foram preparadas em concentração a 5\%. Para a extração do corante natural, utilizou-se água destilada e 10g de amostra do corante. $\mathrm{O}$ extrato foi preparado, com adição de $10 \mathrm{~g}$ de amostra do corante em $100 \mathrm{~mL}$ de água e aquecido até a fervura. As soluções foram preparadas a uma concentração de 5\% (Carvalho Filho \& Pereira, 2021).

\section{Resultados e Discussão}

\section{Descrição das Plantas}

\section{Thunbergia erecta (Thunbergia erecta (Benth.) T. Anderson}

A Thunbergia erecta (Thunbergia erecta (Benth.) T. Anderson) Figura 2, conhecida popularmente como tumbérgiaazul-arbustiva, de caráter ornamental. Thunbergia erecta é uma planta apropriada para plantio isolado, ou em grupos na forma de renques ao longo de muros, muretas, paredes e cercas, a pleno sol ou meia-sombra, cujas flores atraem beija-flores (Souza, 2010., Silveira et al., 2014 \& Lorenzi, 1999). 
Figura 2: Thunbergia erecta (Thunbergia erecta (Benth.) T. Anderson).

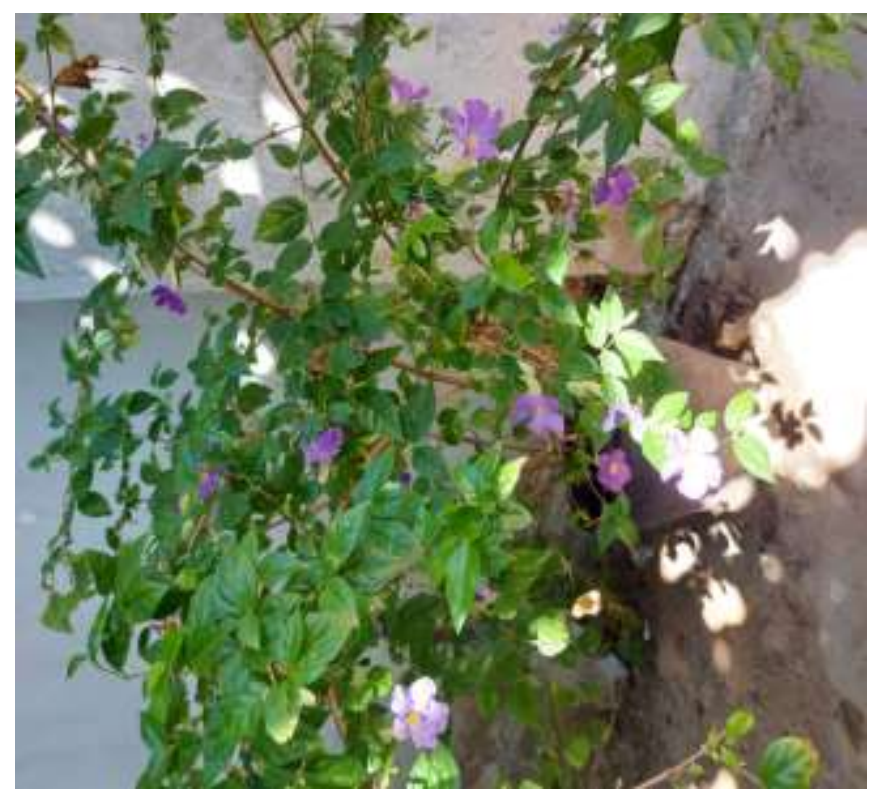

Fonte: Autores (2021).

\section{Chapéu-de-napoleão (Thevetia peruviana (pers.) K. Schum)}

O chapéu-de-napoleão (Figura 3) com nome científico Thevetia peruviana (pers.) K. Schum é popularmente conhecido devido à forma de seus frutos, também chamado com Acaimirim, Auaí-guaçu, Cerbera, Noz-de-cobra, é uma pequena árvore originária da América Central, mas largamente distribuída em todas as regiões tropicais devido ao seu aspecto ornamental e pertencente à família Apocynaceae (Schvartsman, 1979).

Figura 3: Chapéu-de-napoleão (thevetia peruviana (pers.) k. schum).

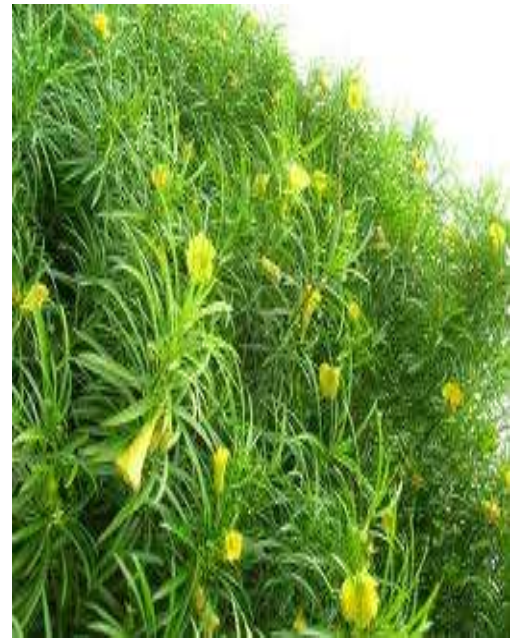

Fonte: Autores (2021).

Esta planta é arbustiva, de textura lenhosa e lactescente (seiva leitosa) com caule único e porte de 3 a 4 metros. As flores são muito bonitas, tubulares, perfumadas, de coloração laranja ou amarela. Ocorrem ainda variedades de flores brancas ou róseas. Os frutos dessa árvore são responsáveis por casos graves de intoxicações, em especial em crianças, que comem suas castanhas. Os principais componentes tóxicos pertencem à classe dos glicosídeos cardiotóxicos (Oliveira et al., 2003). 


\section{Cabeça-de-velho (Euphorbia leucocephala Lotsy)}

A planta cabeçã-de-velho (Euphorbia leucocephala Lotsy) (Figura 4), popularmente conhecido como cabeça de velho, neve da montanha, véu de noiva etc. Apresenta-se como arbusto de textura semi-herbácea, de 2-3 m de altura, de caule marrom-claro, muito ramificado. As flores são brancas, muito numerosas, vistosas, e se formam durante o outono, prolongando-se até o inverno. (Silva \& Lemos, 2002; Carvalho Filho \& Pereira, 2021).

Figura 4: Cabeça de velho (Euphorbia leucocephala Lotsy.).

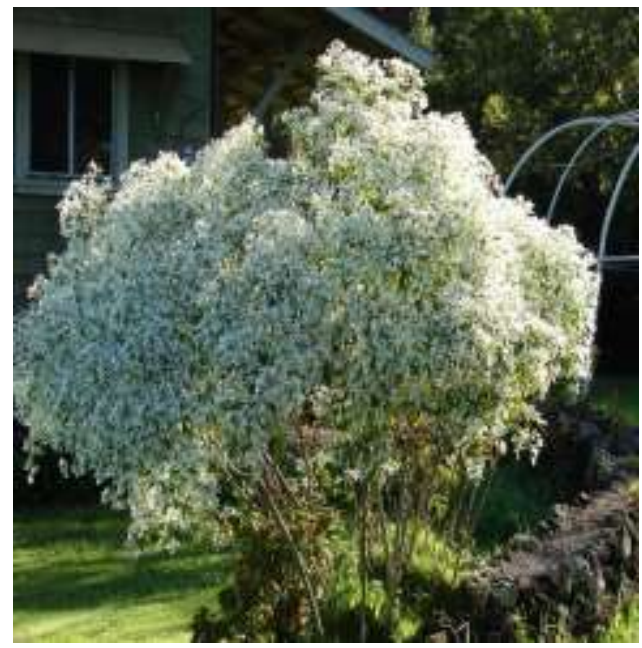

Fonte: Carvalho Filho \& Pereira (2021).

\section{Alamanda-amarela (Allamanda cathartica L.)}

A alamanda (Figura 5) de nome científico Allamanda cathartica L., é conhecida popularmente como alamanda, alamanda-amarela e dedal-de-dama (Lorenzi \& Sousa, 1999). A alamanda floresce, na primavera e no verão. Essa planta é nativa de formações florestais do nordeste e leste do Brasil. (Silva, 2007; Uchôa et al., 2016).

Figura 5: Alamanda-amarela (Allamanda cathartica L).

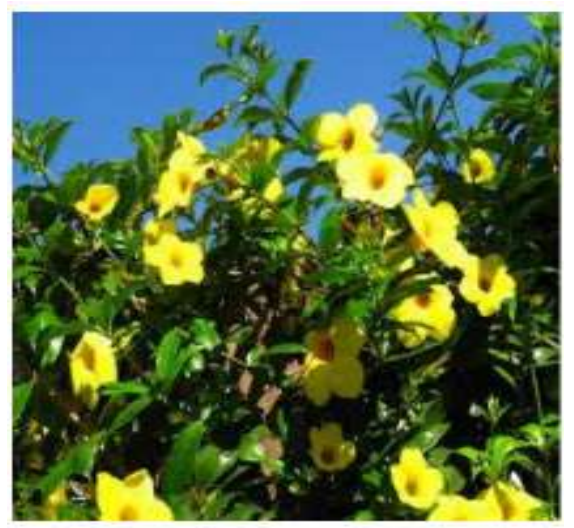

Fonte: Uchôa et al., (2016).

\section{Louro variegado (Codiaeum variegatum L.).}

O louro variegado (Figura 6), de nome científico (Codiaeum variegatum (L.) A. Juss) também conhecido como cróton, cróton de jardim dentre outros, é uma planta da família Euphorbiaceae. Esta planta é um arbusto, grande e 
semilenhosos do Sudeste Asiático, com altura entre 2 e 3m, folhas lactescentes, folhas grandes e coloridas. (Souza \& Lorenzi, 2008; Uchôa et al., 2016).

Figura 6: Louro variegado (Codiaeum variegatum (L.) A. Juss).

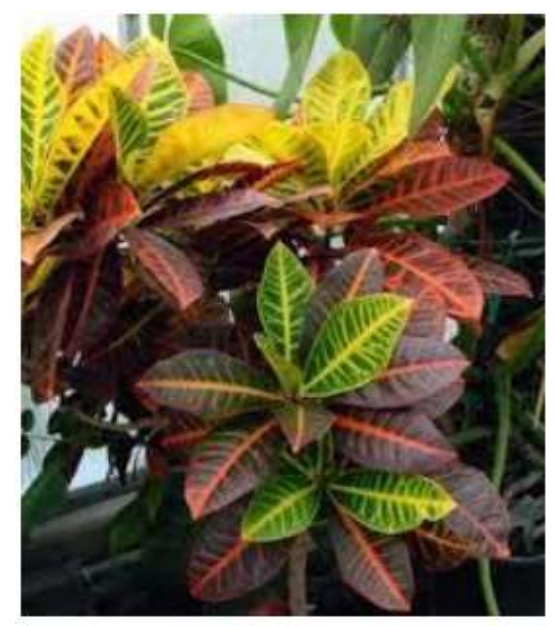

Fonte: Uchôa et al., (2016).

\section{Uso como indicador}

Os corantes naturais (Tabela 1) apresentaram colorações extremas em função do pH. Os corantes $1,3,4$ e 5 apresentaram coloração vermelha em meio ácido e ambos os extratos apresentaram coloração verde em meio básico. O corante 2 em meio ácido, não apresentou variação de cor em função do pH, apresentando semelhança com o indicador sintético fenolftaleína.

Tabela 1: Coloração em meio ácido e meio básico.

\begin{tabular}{|c|c|c|c|c|c|c|c|c|c|}
\hline \multicolumn{2}{|c|}{$\begin{array}{l}\text { Indicador } 1 \\
\text { Thunbergia }\end{array}$} & \multicolumn{2}{|c|}{$\begin{array}{l}\text { Indicador } 2 \\
\text { péu-de-napoleão }\end{array}$} & \multicolumn{2}{|c|}{$\begin{array}{c}\text { Indicador } 3 \\
\text { Cabeça-de-velho }\end{array}$} & \multicolumn{2}{|c|}{$\begin{array}{l}\text { Indicador } 4 \\
\text { Allamanda }\end{array}$} & \multicolumn{2}{|c|}{$\begin{array}{c}\text { Indicador } 5 \\
\text { Cróton }\end{array}$} \\
\hline $\begin{array}{l}\text { Meio } \\
\text { Ácido }\end{array}$ & $\begin{array}{c}\text { Meio } \\
\text { Básico }\end{array}$ & $\begin{array}{l}\text { Meio } \\
\text { Ácido }\end{array}$ & Meio Básico & $\begin{array}{l}\text { Meio } \\
\text { Ácido }\end{array}$ & $\begin{array}{l}\text { Meio } \\
\text { Básico }\end{array}$ & $\begin{array}{l}\text { Meio } \\
\text { Ácido }\end{array}$ & $\begin{array}{l}\text { Meio } \\
\text { Básico }\end{array}$ & $\begin{array}{l}\text { Meio } \\
\text { Ácido }\end{array}$ & $\begin{array}{l}\text { Meio } \\
\text { Básico }\end{array}$ \\
\hline Vermelho & Verde & Incolor & Verde/Amarelo & Vermelho & Verde & Vermelho & Verde & Vermelho & Verde \\
\hline
\end{tabular}

Fonte: Autores (2021).

Observa-se na Tabela 1 que os corantes naturais apresentaram-se como excelentes indicadores ácido-base, devido à capacidade de mudança de coloração em função do pH das soluções, comprovando a eficácia desses corantes em soluções laboratoriais e também em soluções comerciais.

Nos pigmentos (antocianinas), à medida que o pH varia (maior ou menor acidez), elas mudam de cor pelo acréscimo de $\mathrm{OH}^{-}$no carbono 2 (Figura 7) (Soares, Silva \& Cavalheiro, 2001). 
Figura 7: As antocianinas têm cores diferentes a pH mais ácido (à esquerda) ou mais básico (à direita).

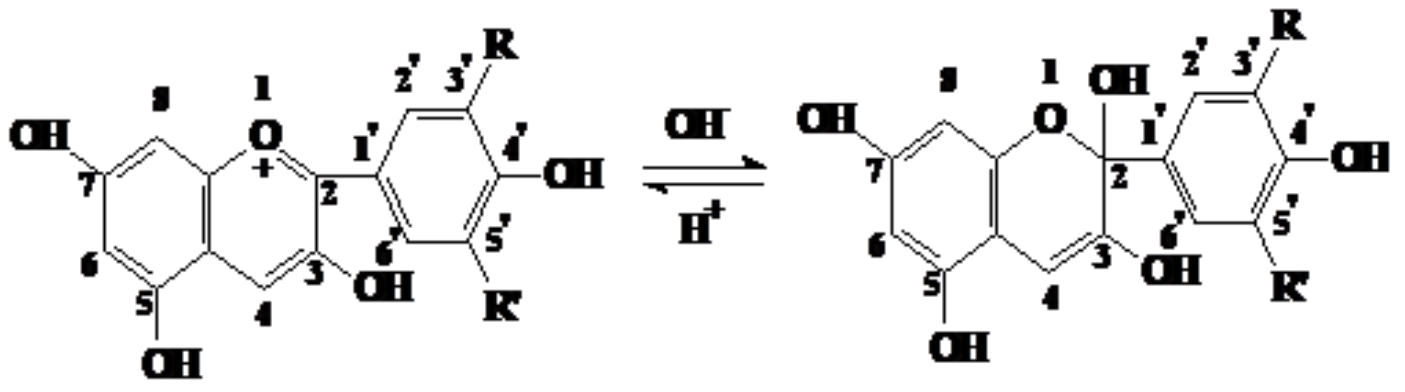

Fonte: Soares, Silva \& Cavalheiro (2001).

As mudanças nas estruturas desses pigmentos ocorrem com a variação do $\mathrm{pH}$, tendo em vista, que essas alterações são responsáveis pelo as colorações diferentes no meio, incluindo o amarelo em meio alcalino alto. Essas alterações podem ser analisadas na Figura 8 (Terci \& Rossi, 2002).

Figura 8: Possíveis mudanças estruturais das antocianinas em meio aquoso em função do pH (Terci \& Rossi, 2002).

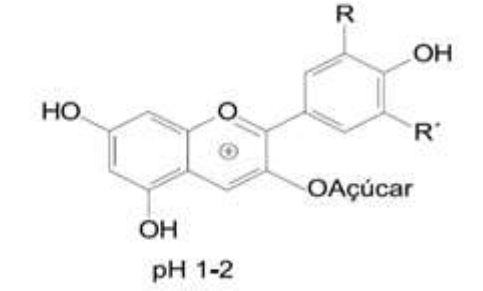

cátion flavilico,vermelho

$$
\begin{gathered}
\mathrm{H}^{+} \\
-\mathrm{H}_{2} \mathrm{O}
\end{gathered} \mid \begin{aligned}
& \mathrm{OH}^{-} \\
& +\mathrm{H}_{2} \mathrm{O}
\end{aligned}
$$
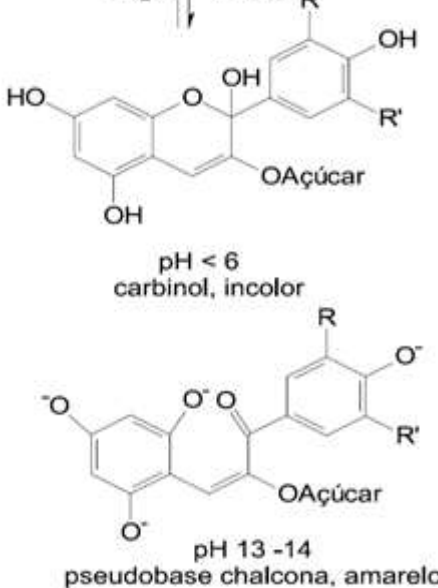
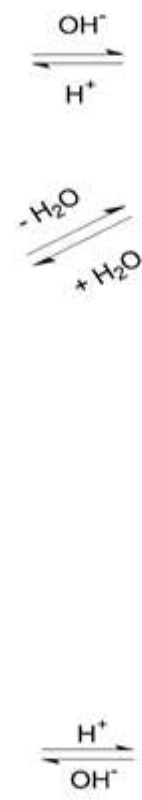

O<smiles>[R]c1cc(-c2oc3cc(=O)cc(O)c-3cc2OC(=O)Cl)cc([R])c1O</smiles>

$$
\mathrm{pH} 6,5-8
$$

anidrobase, violeta<smiles>[R]c1cc(-c2oc3cc(=O)cc(O)c-3cc2OC(=O)Cl)cc([R])c1[O-]</smiles>

$\mathrm{OH}$
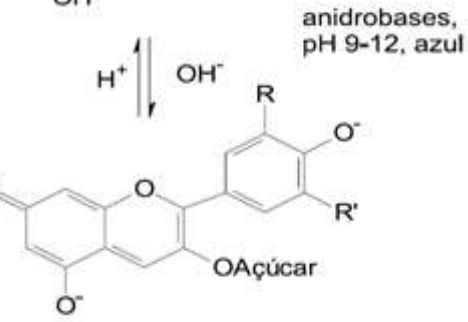

Fonte: Terci \& Rossi (2002)

As mudanças estruturais, permitem alterações de coloração em função do pH do meio em que elas se encontram, portanto, faz com que estes corantes possam ser utilizados como indicadores naturais de pH (Mebane \& Rybolt, 1985). As 
variações estruturais e de coloração presentes na figura 8, são decorrência da variação de pH das soluções em contato com o indicado.

\section{Conclusão}

De acordo com os resultados obtidos, pode-se concluir que os extratos aquosos dos corantes apresentaram excelente variação de coloração em função do $\mathrm{pH}$, podendo ser utilizados como novos indicadores ácido-base para o ensino de Química, uma vez que, essas espécies são de fácil acesso, baixo custo e eficiente com materiais presentes no cotidiano dos alunos, almejando assim, a contribuição das possibilidades de materiais alternativos para o ensino em diversos contextos regionais. Isto pode servir para melhorar o processo de ensino-aprendizagem, vindo a ser concorrente de peso aos indicadores naturais e sintéticos presente na literatura. Essa pesquisa, com o uso de novos corantes pode servir de suporte para trabalhos futuros com a utilização desses pigmentos, uma vez que eles são bem aceitos não só na área da Química, como também na área Alimentícia.

\section{Referências}

Azambuja, C. D., Goi, M. E. J. \& Hartmann, A. M. (2021). A formação docente em química e as práticas pedagógicas dos professores da educação básica: the teaching training in chemistry and the pedagogical practices of teachers of basic education. Contexto \& Amp, 36 (115).

Benite, A. M. C. \& Benite, C. R. M. (2009). O laboratório didático no ensino de química: uma experiência no ensino público brasileiro. Ibero americana de Educación. 48(2), 1-2.

Carvalho Filho, R. S. C. \& Pereira, J. C. A. (2021). Novo indicador natural ácido-base para o ensino de química a partir da euphorbia leucocephala lotsy. ln: Pereira, J. C. O Ensino e a Pesquisa em Química. 1. ed. Ponta Grossa - PR: Atena. https://doi.org/10.22533/at.ed.280212608.

Coelho, A. G. (2011). Estudo da degradação térmica de antocianinas de extratos de uva (Vitis vinifera L. 'Brasil') e jabuticaba (Myrciaria cauliflora). 98p. Dissertação (Mestrado em Química Analítica) - Departamento de Química. Universidade Estadual de Campinas, Campinas.

Couto, A. B., Ramos, L. A. \& Cavalheiro, E. T. G. (1998). Aplicação de pigmentos de flores no ensino de química. Química Nova, 21 (2), $221-227$.

Curtright, R., Rynearson, J. A. \& Markwell, J. (1996). Anthocyanins Model compounds for learning about more than pH. J. Chem Educ, 73 (4), $306-309$.

Di Carlo, G., Mascolo, N., Izzo, A. A. \& Capasso, F. (1999). Review article: Flavonoids old and new aspects of a class of natural therapeutic drugs. Life Sci, $65(4), 337-353$.

Dias, M. V. D., Guimarães, P. I. C. \& Merçon, F. (2003). Corantes Naturais: Extração e emprego como Indicadores de pH. Quimica Nova, $17,27-31$.

Dreosti, I. E. (2000). Antioxidant polyphenols in tea, cocoa, and wine. Nutrition, 692, 7-8.

Harborne, J. B. \& Grayer, R. J. (1998). The anthocyanins. In: GRAYER, R. J. The flavonoids: advances in research since 1980. Chapmam \& Hall, London, 120 .

Favaro, M. M. A. (2007). Extração, estabilidade e quantificação de antocianinas de frutas típicas brasileiras para aplicação industrial como corantes. 102p. Dissertação (Mestrado em Química) - Instituto de Química. Universidade Estadual de Campinas, Campinas.

Lee, J., Durst, R. W. \& Wrolstad, R. E. (2005). Determination of total monomeric anthocyanin pigment content of fruit juices, beverages, natural colorants, and wines by the $\mathrm{pH}$ differential method: Collaborative study. Journal AOAC International, 88 (5), 1269-1278.

Lorenzi, H. \& Sousa, H. M. Plantas ornamentais do Brasil: arbustivas, herbáceas e trepadeiras. (1999). Nova Odessa: Instituto Plantarum. 1092 p. 1999.

Mebane, R. C. \& Rybolt, T. R. Edible acid-base indicators. (1985). J. Chem. Educ, 62 (4), 285 p.

Oliveira, R. B. \& Godoy, S. A. P. (2003). Plantas tóxicas: conhecimento para a prevenção de acidentes. Holos, 1, 64 p.

Penaforte, G. S. \& Santos, V. S. (2014). O ensino de química por meio de atividades experimentais: aplicação de um novo indicador natural de pH como alternativa no processo de construção do conhecimento no ensino de ácidos e bases. Educamazônia, 8,(2), 8-21.

Schavartsman, S. (1979). Plantas venenosas. Sarvie, 176 p.

Skoog, D. A., West, D. M., Holler, F. J. \& Crouch, S. R. (2015). Fundamentos de Química Analítica. (9. ed.). Cengage Learning.

Silva, D. B. \& Lemos, B. S. Plantas de área verde da Super Quadra Norte. (2002). Embrapa, Brasília, Brasil, 147 p. http://www.alice.cnptia.embrapa.br/bitstream/doc/184370/1/liv003.pdf.

Silva, K. A. B. S. Caracterização dos efeitos do Plumerídeo, um iridóide isolado de Allamanda cathartica L. (Apocynaceae), em modelos de inflação e dor. (2007). Disertação (Mestrado em Farmacologia) - Programa de Farmacologia. 
Research, Society and Development, v. 10, n. 12, e154101220315, 2021

(CC BY 4.0) | ISSN 2525-3409 | DOI: http://dx.doi.org/10.33448/rsd-v10i12.20315

Silva, W. A., Moura, F. J. A., Sousa, J. L. S. \& Correia, J. M. (2020). A utilização do indicador natural para a aplicação de uma atividade experimental no ensino de química. Brazilian Journal of development, 6(4), 16859-16871.

Siveira, A., et al. Plantas ornamentais em quintais urbanos de Rio Branco, Brasil. (2014). Bol Mus Para Emílio Goeldi Cienc Hum, 9(3): 797-813.

Soares, M. H. F. B., Silva, M. V. B., Cavalheiro, E. T. G. Aplicação de corantes naturais no ensino médio. (2001). Eclet. Quím, 26, 98-103.

Souza, C. C. V. Etnobotânica de quintais em três comunidades ribeirinhas na Amazônia Central, Manaus - AM. (2010). 103p. Dissertação de Mestrado Ciências Biológicas - Botânica. Instituto Nacional de Pesquisas da Amazônia - INPA, Manaus.

Souza, V. C., Lorenzi, H. (2008). Botânica sistemática. Instituto Plantarum, 2 ed.

Terci, D. B. L. \& Rossi, A. V. (2002). Indicadores naturais de pH: usar papel ou solução?. Quimica Nova, 25(4), 684-688.

Uchôa, V. T., Carvalho Filho, R. S. M., Lima, A. M. M., \& Assis, J. B. (2016). Utilização de plantas ornamentais como novos indicadores naturais ácido-base no ensino de química. Holos, 32(2), 152-165. 